\title{
Enhanced Scope of a Phase-2 CMS Detector for Physics beyond the Standard Model at the High- Luminosity LHC
}

\author{
Kerstin Hoepfner ${ }^{1}$ on behalf of the CMS collaboration \\ RWTH Aachen, III. Phys. Inst. A \\ Otto-Blumenthal-Str., Aachen, Germany \\ E-mail: hoepfnerephysik.rwth-aachen.de
}

\begin{abstract}
To extend the LHC physics program, it is foreseen to operate the LHC in the future with an unprecedented high luminosity. To maintain the experiment's physics potential in the harsh environment of this so-called Phase-2, the detector will be upgraded. The silicon tracker and the forward detectors will have to be replaced to cope with radiation damage. At the same time the detector acceptance will be extended and new features such as a L1 track trigger will be implemented. Simulation studies evaluated the performance of the new, proposed detector components and the impact on representative physics channels. In case of searches for new physics, these studies also shape the future research program. The sensitivity to find new physics beyond the standard model (SM) is significantly improved and will allow to extend the reach for heavy vector bosons, for SUSY, dark matter and exotic long-lived signatures, to name a few.
\end{abstract}

EPS-HEP2017, European Physical Society conference on High Energy Physics

5-12 July 2017, Venice, Italy

\footnotetext{
${ }^{1}$ Speaker
} 


\section{What means high luminosity?}

This paper presents physics studies for the future operation of the LHC collider with highluminosity (HL-LHC), also called LHC Phase-2. High luminosity means about five times higher instantaneous luminosity, with $40 \mathrm{MHz}$ operation (one BX every $25 \mathrm{~ns}$ ) at $\sqrt{ } s=14 \mathrm{TeV}$. This yields challenging conditions for the CMS detector: up to 200 overlaying (pileup) events, high rates and high radiation levels, especially in the forward regions. The total accumulated luminosity is anticipated to reach $3000 / \mathrm{fb}$ of data, corresponding to $10 \mathrm{x}$ the data expected at the end of Phase-1. This environment offers great physics opportunities: e.g. discover new particles (e.g. dark matter, heavy resonances, the ,unexpected"), access weak couplings and rare processes. If discovered, particle properties (mass, couplings, spin, etc.) will be studied.

\section{Ensure detection of unusual signatures}

Since it is not known how new physics will look like, it is essential to be sensitive to all possible signatures. New physics could manifest itself with a non-standard signature, requiring dedicated trigger and reconstruction algorithms and specific detector features. One example (see Fig.1left) are displaced muons from decays of long-lived particles (e.g. with large impact parameter $\mathrm{d}_{\mathrm{xy}}$ ) that need a stand-alone muon trigger without vertex constraint. Another example is slow moving particles (see Fig.1-right) which deposit anomalous (large) dE/dx in the silicon tracker. This feature has to be kept for Phase-2 as such a signature allows efficient separation of these special particles from the MIP background.
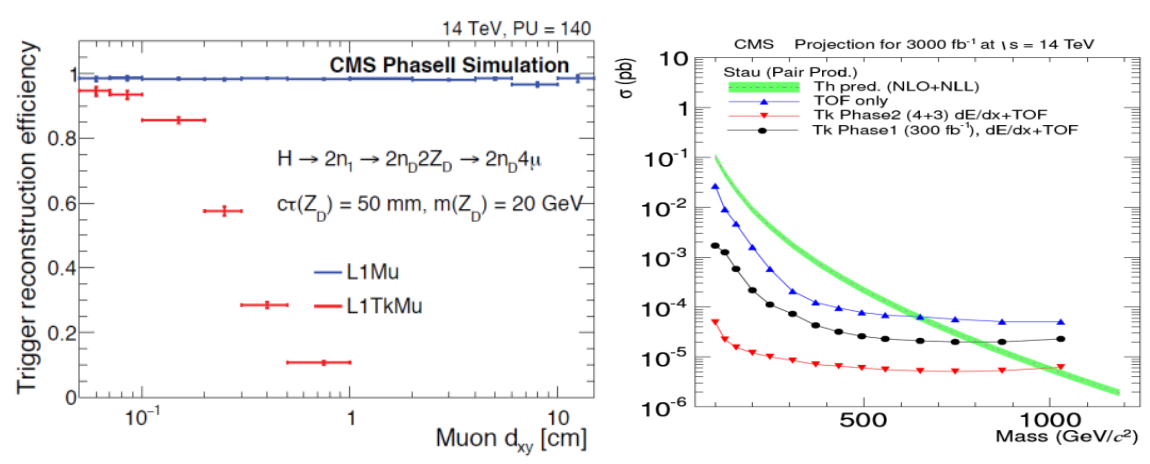

Fig. 1: Ensure detection of unusual signature demonstrated on two examples. Left: To trigger displaced muons with large impact parameter $d_{x y}$ needs a stand-alone muon trigger without vertex constraint. Right: Sensitivities for heavy stable charged particles in different scenarios.

\section{Search for dark matter in the jet + MET final state}

One of the top priorities of the HL-LHC is the search and/or characterization of dark matter (DM). A classical benchmark channel among the many DM collider searches is the jet + MET final state, where the MET is caused by the pair of DM particles recoiling against a jet. Following the recommendations of LHC DM forum [arXiv: 1507.00996] the model is described by four parameters $-M_{m e d}, M_{D M}, g_{S M}$, $g_{D M}$. Exclusion limits are projected to HL (see Fig. 2) for an axial vector and a pseudoscalar mediator using the parametrized DELPHES simulation [4] with the run-2 results [arXiv:1703.01651] as baseline. Besides the present knowledge of systematic uncertainties, two plausible scenarios with reduced systematics are tested. The key is the understanding of MET: very high MET is dominated by the size of control samples and background extrapolation, low MET is systematics dominated. 

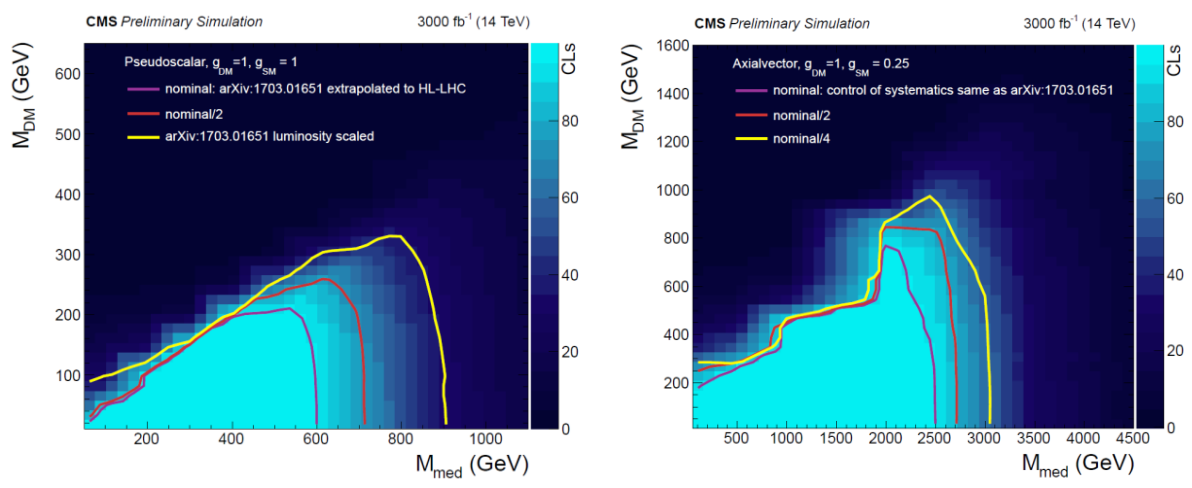

Fig. 2: Projected exclusion limits for a DM search in the jet+MET final state, for axial vector (left) and pseudoscalar (right) mediators. Scenarios of systematics and DM parameters are explained in the text.

\section{Projected sensitivities for heavy gauge bosons}

New heavy bosons (neutral as $\mathrm{Z}^{\prime}$, charged as $\mathrm{W}^{\prime}$ ) are predicted in many SM extensions. Couplings to third family may be enhanced, giving special emphasis to final states containing top and/or bottom quarks. Fig. 3 shows extrapolations of existing run-2 analyses to HL statistics considering scenarios of systematic uncertainties as shown in the figure legend. The decay $\mathrm{W}^{\prime} \rightarrow$ tb $\rightarrow \mathrm{e} / \mu+\mathrm{b}$-jets allows, for example, to probe scenarios such as $\mathrm{m}\left(v_{\mathrm{R}}\right)>\mathrm{m}\left(\mathrm{W}^{\prime}{ }_{\mathrm{R}}\right)$, which cannot be studied with leptonic $\mathrm{W}^{\prime}$ searches. For $\mathrm{Z}^{\prime}$ two distinct channels are projected, either with lepton+jets in the final state or fully hadronic. The knowledge of systematic uncertainties has a visible impact on the physics reach. One expects improvements on the theoretical knowledge of higher order corrections, while detector understanding and data-driven methods will profit from larger statistics.
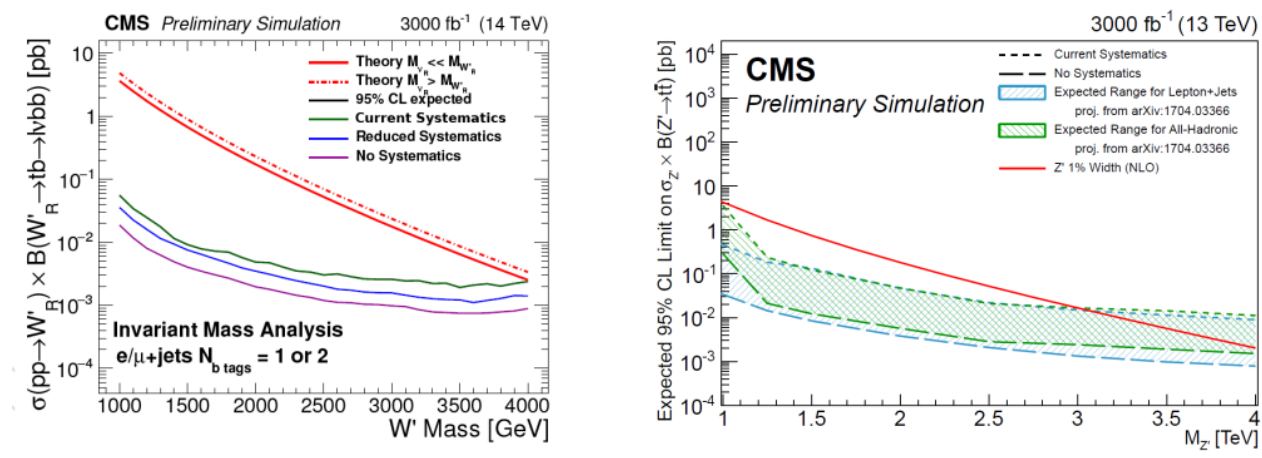

Fig. 3: Projected exclusion limits from run-2 analyses with different systematics scenarios Left: $W^{\prime} \rightarrow t b$ $\rightarrow e / m u+b$-jets (based on CMS-PAS-B2G-16-017). Right: $Z^{\prime} \rightarrow t t \rightarrow e / \mu+$ jets ( $b$ - or $t$-tagged jet) (based on CMS-PAS-B2G-15-002) and $Z^{\prime} \rightarrow t t \rightarrow$ all hadronic (extrapolated from CMS-PAS-B2G-15-003).

\section{General References:}

[1] CMS-PAS-EXO-14-007

[3] CMS-TDR-15-001, CDS1994307 (GE1/1 TDR)

[2] CMS-PAS-FTR-16-005

[4] DELPHES simulation, arXiv:1307.6346 